\title{
EDITORIAL
}

\section{Cardiovascular genetics}

Journal of Human Genetics (2016) 61, 1; doi:10.1038/jhg.2015.147

$\mathrm{C}$ ardiovascular diseases represent the major cause of mortality and morbidity occupying a considerable part of medical expenses in the world. There are important and urgent social needs to overcome the disorders by elucidating molecular etio-pathological mechanisms to establish a bridgehead for developing novel strategic medicine including new drugs, new therapeutic devices or new biomarkers, as well as novel preventive tactics. For this end, genetics has played a potent role mainly because of its nature, hypothesis-free methodology. Indeed, genetic investigation has been powerful in identifying responsible genes for the cardiovascular diseases, of which involvement in the pathogenesis has never been predicted by their protein function. With the use of recent ultra-rapidly developed technologies in acquiring and handling data on genetic information, which can be achieved much faster, much cheaper and much larger in scale than ever before, hundreds of genes that are responsible for or associated with the cardiovascular diseases have been identified. Another important aspect on the cardiovascular disorders arises at the developmental stage of the heart, when abnormalities in specific genes, regulation of gene expression or non-coding regulatory genome were found to have crucial roles in the pathogenesis. In addition, it is worth noting that several fruits in this research field have already been served on the tables in clinical fields as diagnostic or assessment tools; pharmacogenomics is one of the front runners in the clinical stage.

As handling editors of this special issue, we are very proud to offer readers of the Journal of Human Genetics 11 thought-provoking review articles and 1 commentary that are focused on the cardiovascular genetics. These articles cover wide range of topics in the cardiovascular genetics field as described above, and we are confident that they show both the current state of evidence and potential insights into future directions in this attractive and practical field.

Toshihiro Tanaka ${ }^{1,2,3}$ and Akinori Kimura ${ }^{4,5}$

${ }^{1}$ Laboratory for Cardiovascular Diseases, RIKEN Center for Integrated Medical Sciences, Yokohama, Japan; ${ }^{2}$ BioResource Research Center, Tokyo Medical and Dental University (TMDU), Tokyo, Japan; ${ }^{3}$ Department of Human Genetics and Disease Diversity, Graduate School of Medical and Dental Sciences, Tokyo Medical and Dental University (TMDU), Tokyo, Japan; ${ }^{4}$ Department of Molecular Pathogenesis, Medical Research Institute, Tokyo Medical and Dental University (TMDU), Tokyo, Japan and ${ }^{5}$ Department of Genetic Regulation, Graduate School of Medical and Dental Sciences, Tokyo Medical and Dental University (TMDU), Tokyo, Japan E-mail: ttana.brc@tmd.ac.jp (TT) and akitis@mri.tmd.ac.jp (AK) 